\title{
Differential occurrence of S100A7 in breast cancer tissues: A proteomic-based investigation
}

\author{
Patrizia Cancemi ${ }^{1,2}$, Gianluca Di Cara ${ }^{2}$, Nadia Ninfa Albanese ${ }^{2,3}$, Francesca Costantini ${ }^{1}$, \\ Maria Rita Marabeti ${ }^{1}$, Rosa Musso ${ }^{1}$, Ignazio Riili ${ }^{4}$, Carmelo Lupo ${ }^{4}$, Elena Roz ${ }^{4}$ and \\ Ida Pucci-Minafra² \\ ${ }^{1}$ Dipartimento Biomedico di Medicina interna e specialistica (Dibimis) sezione Oncologia Sperimentale e \\ Applicazioni Cliniche, Università di Palermo, Italy \\ ${ }^{2}$ Centro di Oncobiologia Sperimentale (COBS), at “La Maddalena" D.O. III livello, Palermo, Italy \\ ${ }^{3}$ Dipartimento di Fisica, Università di Palermo, Italy \\ ${ }^{4}$ Ospedale "La Maddalena" D.O. III livello, Palermo, Italy
}

Purpose: The present study reports for the first time a large-scale proteomic screening of the occurrence, subcellular localization and relative quantification of the S100A7 protein among a group of 100 patients, clinically grouped for the diagnosis of infiltrating ductal carcinoma (IDC). Experimental design: To this purpose, the methods of differential proteomics, Western blotting, and immunohistochemistry were used.

Results: The identity of two isoforms of the protein was assessed by mass spectrometry and immunologically confirmed. Moreover, we proved by immunocytochemical applications the exclusive localization of the protein within the neoplastic cells. The correlation of S100A7 expression levels with the collective profile of cancer patients' proteomics predicted functional interactions, distinct for the two isoforms. The S100A7b isoform was significantly correlated with specific protein clusters (calcium binding, signaling and cell motion, heat shock and folding) and intercrossing pathways (antioxidant, metabolic and apoptotic pathways), while the more acidic isoform was correlated with a narrow number of proteins mainly unrelated to the $b$ isoform. Conclusions and clinical relevance: This study is the first proteomic-based report on S100A7 in a large series of IDC patients. The correlation with in silico data may significantly contribute the knowledge of possible pathways for S100A7, providing novel insights into the mechanism of action of this protein. We suggest that each S100A7 isoform is involved in critical phases of the breast cancer growth and progression, probably through interaction with different partner proteins.

Keywords:

Breast cancer / S100A7

\section{Introduction}

S100A7 protein, also named Psoriasin, is a member of the EF-hand superfamily of S100-calcium-binding proteins, so called because of their solubility in a saturated solution of ammonium sulphate. The first member of this family was identified in the nervous system in 1965 [1].

Correspondence: Dr. Patrizia Cancemi, Centro di Oncobiologia Sperimentale (COBS), at "La Maddalena" D.O. III livello, Via San Lorenzo Colli, 312, Palermo 90146, Italy

E-mail: patrizia.cancemi@unipa.it

Fax: +39-091-6806420

Abbreviations: IDC, infiltrating ductal carcinoma; IHC, immunohistochemistry
Received: August 10, 2011

Revised: March 9, 2012

Accepted: April 12, 2012
Presently, the known members of the S100 protein family in humans are at least 25 , differentially expressed in tissues and cells, suggesting the existence of diversified functions of the family members according to given phenotypes [2]. Most of them form homo- or heterodimers and some oligomers, in the presence or absence of calcium [3]. The repertory of S100 protein functions covers several biological processes, through a broad range of intracellular and extracellular activities $[4,5]$. Intracellular functions include control and/or regulation of calcium homeostasis, protein phosphorylation, cell cycle and growth control, cell differentiation, cytoskeleton reorganization and motility, and transcriptional activities $[5,6]$. Extracellularly, they act in a cytokine like manner

Colour Online: See the article online to view Fig. 4 in colour. 
through a transmembrane receptor of the immunoglobulin superfamily named RAGE (receptor for advanced glycation endproducts) [7].

In consideration of the critical roles played by S100 proteins in such large spectrum of biological activities, it is not surprising that improper expression of S100 members is correlated with many pathologies and especially with cancer. A number of S100 proteins have been shown to interact with oncogenes and various proteins involved in cancer progression, including $\mathrm{p} 53$, cytoskeletal proteins, matrix metalloproteinases (MMPs), and others, either in calcium-dependent or calcium-independent ways [8]. Moreover, there are also several evidences that overexpression of S100 proteins occurs in cancer, for which reason it is proposed their clinical application for the diagnosis and staging of human tumors, as well as for prediction of prognosis $[9,10]$.

In a previous study, we have described a large-scale proteomic investigation performed on breast cancer patients for the screening of multiple forms of S100 proteins [11].

The results have shown that some $\mathrm{S} 100$ protein members were ubiquitously expressed in almost all patients, while others appeared more sporadic among the same group, without apparent correlation with the clinical parameters. Most of the detected S100 members appeared reciprocally correlated. Among the sporadic forms of S100 proteins, the S100A7 was one of the prominent, also for the high level of relative concentration reached in many cases.

S100A7 was originally identified as a protein overexpressed in psoriatic keratinocytes [12], since then it has been found overexpressed in several carcinomas, including skin [13], oral squamous cell carcinoma [14], esophagus [15], bladder [16], lung [17], as well as adenocarcinomas of the stomach [18] and the breast [19-23].

Psoriasin was found to be secreted but also present in the cytoplasm and the nucleus of cells expressing it. Secreted S100A7 may exert a chemotactic potential [24] and more recently, it has been implicated in inflammation with an antibacterial action [25]. Meanwhile, S100A7 is also believed to be associated with several intracellular proteins such as RanBPM [26], epidermal fatty acid binding protein [27], and Jab-1 [28].

However, the knowledge on the involvement S100A7 in cancer is based mostly on in vitro models and this is a limitation due to the difficulty of conducting basic research on human biopsies. In the present study, our aim was to add information to the in vivo expression of the S100A7 by: (i) determining the frequency of its occurrence in a large series of ductal infiltrating surgical tissues; (ii) to detect its localization within the tumor tissues; (iii) to ascertain its possible relationships to protein clusters generated by differential proteomic profiling on the patient's maps. With this purpose, the methods of differential proteomics, MALDI-TOF spectrometry, Western blotting, and immunohistochemistry (IHC) were used.

Our results confirmed the sporadic occurrence of S100A7 among patients and showed that, when present, the protein is primarily expressed in breast cancer cells and not in the unaffected glandular structures or in the mesenchymal cells. Moreover, the existence of two isoforms of S100A7, arbitrarily named $a$ and $b$, with different levels of silver-stained intensity among patients, was noticed.

Statistical applications, to explore possible correlations of $\mathrm{S} 100 \mathrm{~A} 7 a$ and $b$ expression levels with patient proteomics, showed a diversified pattern for the two isoforms.

The S100A7b isoform was significantly correlated with a broad range of proteins involved in cytoplasmic activities (calcium binding, signaling and cell motion, heat shock and folding) and crossing pathways (antioxidant, metabolism, regulation of apoptosis). On the contrary, S100A7 $a$ was correlated with a limited number of apparently unrelated proteins, six of which also unrelated to the $b$ isoform and with key roles in cancer progression.

\section{Materials and Methods}

\subsection{Clinical specimens}

Aliquots of breast cancer and its adjacent nontumoral tissues were obtained during surgical intervention between 2003 and 2007 in the Breast Unit of the La Maddalena Hospital and immediately frozen at $-80^{\circ} \mathrm{C}$ until used. Research was carried out in compliance with the Helsinki Declaration with the patients' written consent and with the approval of the Institutional Review Board ( $\left.\mathrm{N}^{\circ} 515 / 2008\right)$ from the La Maddalena Hospital. The patients of this study did not receive any cytotoxic/endocrine treatment prior to surgery. Diagnosis of ductal breast cancer $(\mathrm{G} 2 / \mathrm{G} 3)$ was confirmed histopathologically.

\subsection{Sample preparations}

Sample preparation and analysis was carried out essentially as described in [11]. The frozen breast tissue samples were washed several times with PBS and homogenized in RIPA buffer (50 mM Tris pH 7.5, 0.1\% Nonidet P-40, 0.1\% deoxycholate, $150 \mathrm{mM} \mathrm{NaCl}, 4 \mathrm{mM}$ EDTA), containing a mixture of protease inhibitors $(0.01 \%$ aprotinin, $10 \mathrm{mM}$ sodium pyrophosphate, $2 \mathrm{mM}$ sodium orthovanadate, $1 \mathrm{mM}$ PMSF). The extraction was carried out overnight at $4^{\circ} \mathrm{C}$ with the same buffer. The total cellular lysate was centrifuged to remove tissue debris, and the resulting supernatant dialysed against ultrapure distilled water, lyophilized and stored at $-80^{\circ} \mathrm{C}$ until use. The total protein concentration was determined by the Bradford method using bovine serum albumin as a standard.

\subsection{D-IPG}

The proteins extracted from breast cancer tissues and normal adjacent tissues were solubilized in a buffer containing $4 \%$ CHAPS, $40 \mathrm{mM}$ Tris, $65 \mathrm{mM}$ DTE in $8 \mathrm{M}$ urea. Aliquots of 
$45 \mu \mathrm{g}$ (analytical gels) or $1.5 \mathrm{mg}$ (preparative gels) of total proteins were separately mixed with $350 \mu \mathrm{L}$ of rehydration solution containing $8 \mathrm{M}$ urea, 2\% CHAPS, $10 \mathrm{mM}$ DTE, and $0.5 \%$ carrier anpholytes (Resolyte 3.5-10), and applied for IEF using commercial sigmoidal IPG strips, $18-\mathrm{cm}$ long with $\mathrm{pH}$ range 3.0-10. The second dimension was carried out on 9-16\% linear gradient polyacrylamide gels (SDS-PAGE), and the separated proteins were visualized by ammoniacal silver staining.

\subsection{Image acquisition and data analysis}

Silver stained gels were digitized using a computing densitometer and analyzed with Image Master software (Amersham Biosciences, Uppsala, Sweden).

Quantitative expression levels were calculated as the volume of the spots (i.e., integration of optical density over the spot area). In order to correct for differences in gel staining, spot volumes relative to the sum of the volume of all spots on each gel (\%Vol) were calculated by the software. Measurements of relative expression levels of individual protein spots were normalized in each map for actin content (N\%V) [29].

For statistical analyses Ms Excel and Graph Pad Prism 4 software were used. Correlation of S100A7 protein with the collective profile of cancer patients proteomics was performed using the Pearson correlation test. $p<0.05 *$ was considered significant, $p<0.01 * *$ was considered highly significant, $p<$ $0.001 * * *$ was considered very highly significant.

\subsection{Protein identification}

$\mathrm{N}$-Terminal microsequencing was performed by automated Edman degradation in a protein sequencer (Procise, 419 Applied Biosystems, Monza, Milano, Italy), as previously described [30]. Mass spectrometric sequencing was performed by Voyager DE-PRO (Applied Biosystems) mass spectrometer as described [31]. Briefly, proteins were digested using sequencing-grade trypsin $(20 \mu \mathrm{g} / \mathrm{vial})$. The tryptic peptide extracts were dried and redissolved in $10 \mu \mathrm{L}$ of $0.1 \%$ TFA. The matrix, CHCA, was purchased from Sigma-Aldrich, St. Louis, MO, USA. A saturated solution of CHCA $(1 \mu \mathrm{L})$ at $2 \mathrm{mg} / 200 \mu \mathrm{L}$ in $\mathrm{CH}_{3} \mathrm{CN} / \mathrm{H}_{2} \mathrm{O}(50: 50(\mathrm{v} / \mathrm{v}))$ containing $0.1 \%$ TFA was mixed with $1 \mu \mathrm{L}$ of peptide solution on the MALDI plate and left to dry. MALDI-TOF mass spectra were recorded in the 500-5000 Da mass range, using a minimum of 100 shots of laser per spectrum. Delayed extraction source and reflector equipment allowed sufficient resolution to consider $\mathrm{MH}+$ of monoisotopic peptide masses. Internal calibration was done using trypsin autolysis fragments at $\mathrm{m} / \mathrm{z}$ 842.5100, 1045.5642, and 2211.1046 Da. Peptide mass fingerprinting was compared to the theoretical masses from the Swiss-Prot or NCBI sequence databases using Mascot http://www.matrixscience.com/. Typical search parameters were as follows: $50 \mathrm{ppm}$ of mass tolerance, carbamidomethy- lation of cysteine residues, one missed enzymatic cleavage for trypsin, a minimum of four peptide mass hits was required for a match, and methionine residues could be considered in oxidized form.

\subsection{Western blotting}

For immune detection, the $1 \mathrm{D}$ or $2 \mathrm{D}$ gels were electrotransferred onto nitrocellulose membrane (HyBond ECL, Amersham Biosciences) and stained with Ponceau S (Sigma Aldrich). The membranes were then probed with monoclonal anti-S100A7 (Santa Cruz, Heidelberg, Germany; 1:1000). Following incubation with the peroxidase-linked mouse antibody, the reaction was revealed by the ECL detection system, using high-performance films (Hyperfilm ECL, Amersham Biosciences).

\subsection{Immunohistochemistry}

Sections of 3- $\mu \mathrm{m}$ thick of formalin-fixed, paraffin-embedded surgical breast specimens were deparaffinized in xylene and rehydrated with distilled water. After antigen retrieval in citrate buffer, staining was performed using the BenchMark automated staining system (Ventana Medical System, Tucson, AZ, USA) with monoclonal S100A7 antibody (Santa Cruz; 1:500). Signal was detected with EnVision ${ }^{\circledR}$ Plus SystemHRP (Dako UK, Ely, UK), and visualized with diaminobenzidine. Slides were then counterstained in Mayer's hematoxylin for $10 \mathrm{~s}$, dehydrated in graded alcohol, and mounted. Negative controls, where primary antibody was omitted, were included.

\subsection{Bioinformatics}

DAVID (the databases for annotation, visualization, and integrated discovery) was used for functional analysis. DAVID bioinformatics (http://david.abcc.ncifcrf.gov/) were released in 2003 and extract biological features associated with gene/protein lists. The current version of DAVID not only keeps a gene-GO term enrichment analysis algorithm, but increase over 40 annotation categories with extended annotation content coverage, classifying the gene list into functionally related gene groups.

\section{Results}

\subsection{Proteomics and immunological validation of S100A7}

Figure 1 shows the miniatures of 100 proteomic maps of breast cancer tissue extracts used in the current research, each displaying an individual protein signature, for intensity and number of detectable protein spots, under the same protein loading. The number on each map corresponds to a selected patient. A reference synthetic gel was thereby 


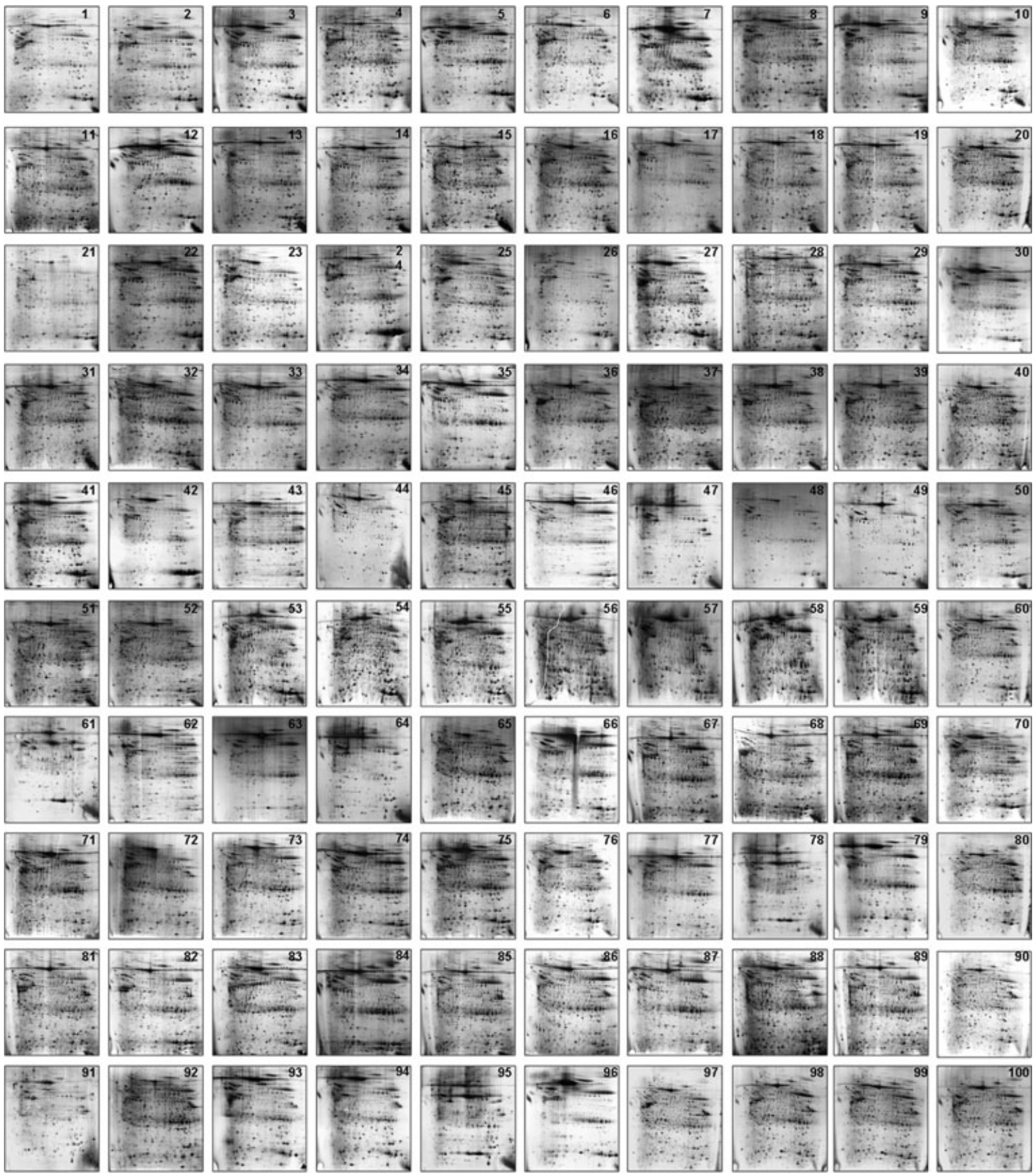

Figure 1. Miniatures of 100 proteomic maps of breast cancer tissue extracts used in the current research. The number on the right corner of each map corresponds to a selected patient.

generated after spot pairs have been determined for each of the 100 maps' collection, according to the Image Master software directions. Figure 2 shows the synthetic gel image, as a reference infiltrating ductal carcinoma (IDC) map, in which only identified proteins are imported and labeled according to the Swiss-prot accession number. The two spots with joined AC number P31151 correspond to the two isoelectric forms of the S100A7 protein. The more prominent displays MW of $11042 \mathrm{Da}$ and $\mathrm{pI}$ of 6.0, which represent the closest expected values of the protein (11 470-6.27), while the second spot, 


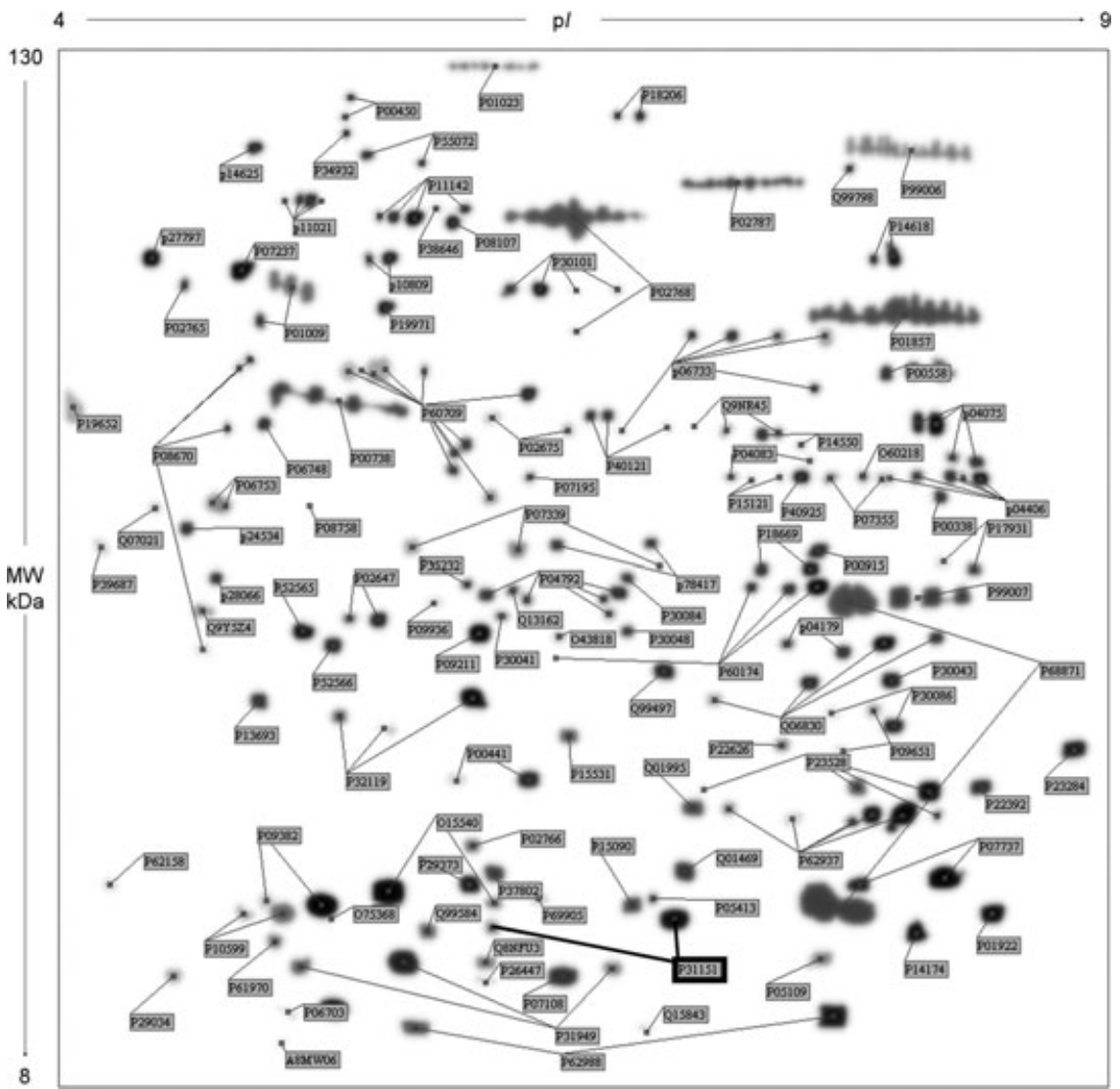

Figure 2. Synthetic gel image, used as a reference IDC map, in which only identified proteins are imported and labeled according to the Swiss-prot accession number. The two spots with joined AC number P31151 (marked in bold) correspond to the two isoelectric forms of the S100A7 protein. usually less intense than the former, is focused in a more acidic $\mathrm{p} I$ (5.35).

To validate these results, we performed parallel 2D-IPG separations followed, respectively, by silver staining and Western blot assays, using the monoclonal anti-S100A7 antibody. The Fig. 3A and B show two gel windows containing the two S100A7 isoforms stained with silver (A), and revealed by western, respectively. An optimal correspondence of the protein-spot intensity levels with both different revelation systems was observed.

\subsection{Spectrometric analysis of the two S100A7 isoforms}

The Fig. 4 reports the list of the most prominent $m / z$ values identified by the MALDI-TOF spectrometry and sequence coverage of the S100A7 $a$ and $b$, respectively. As shown, the peptide mass fingerprinting of the isoform $b$ covers about $95 \%$ of the theoretical masses from the Swiss-Prot sequence database. On the contrary, coverage of the isoform $a$ is of about $60 \%$ of the protein. The unmatching portion of the S100A7a contains part of a putative EF-hand domain, part of a putative calcium-binding domain and two zinc-binding residues.
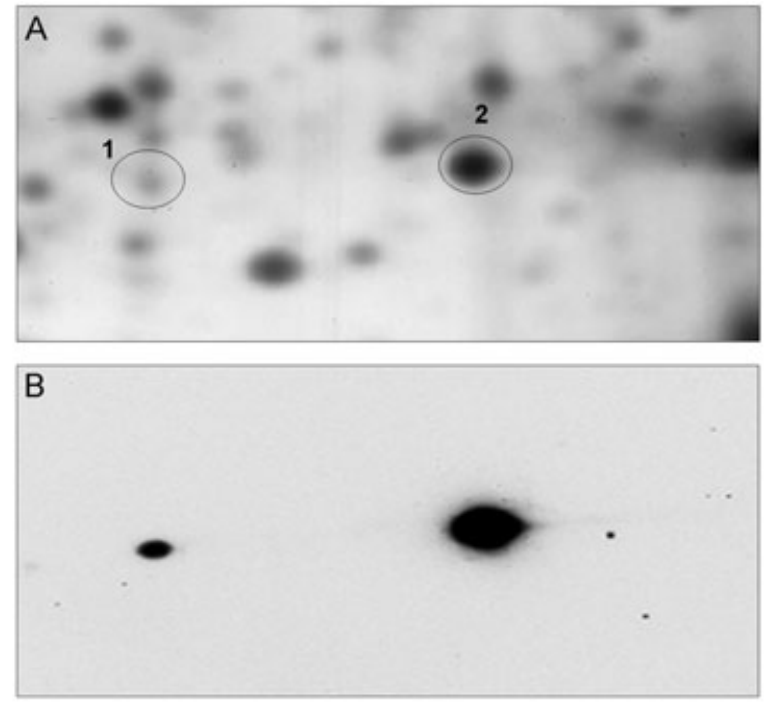

Figure 3. Panel showing two gel windows from 2D-IPG comprising an area covering a $\mathrm{p} / \mathrm{kDa}$ range of 5.5-6.5/12-9 $\mathrm{kDa}$, in which the S100A7 protein spots are focused. The protein spots are stained with silver (A), and revealed by Western blot with anti-S100A7 antibody (B), respectively. 


\section{S100A7 a}

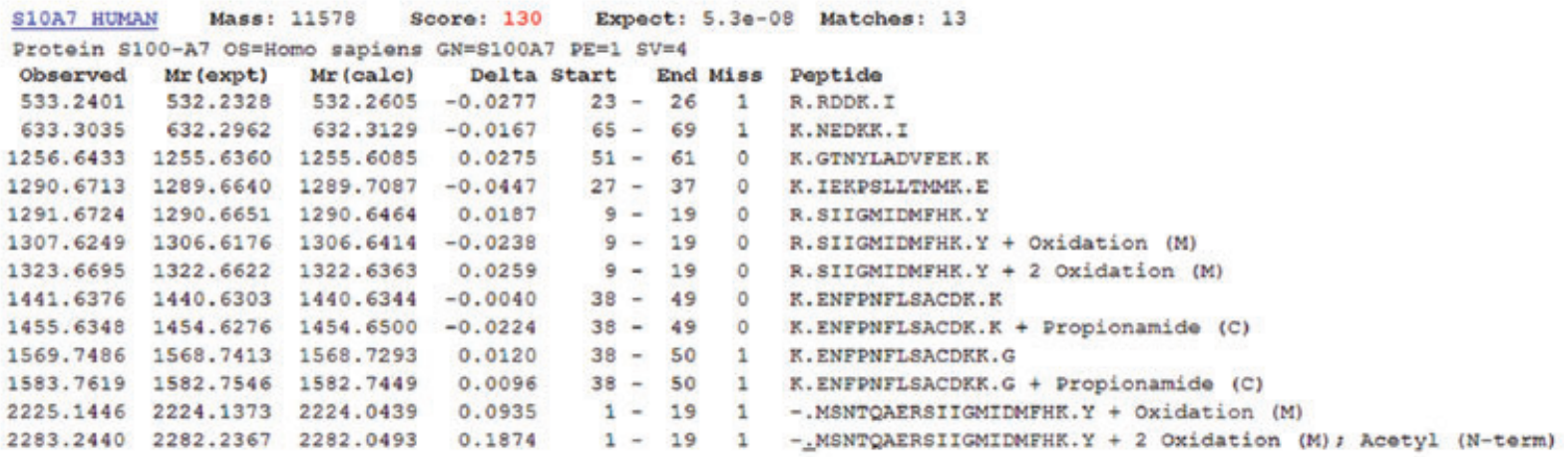

Sequence coverage

1 MSNTQAERSI IGMIDMFHKY TRRDDKIEKP SLLTMMKENF PNFLSACDKK

51 GTNYLADVFE KKDKNEDKKI DFSEFLSLLG DIATDYHKQS HGAAPCSGGS

$101 \mathrm{Q}$

\section{S100A7 b}

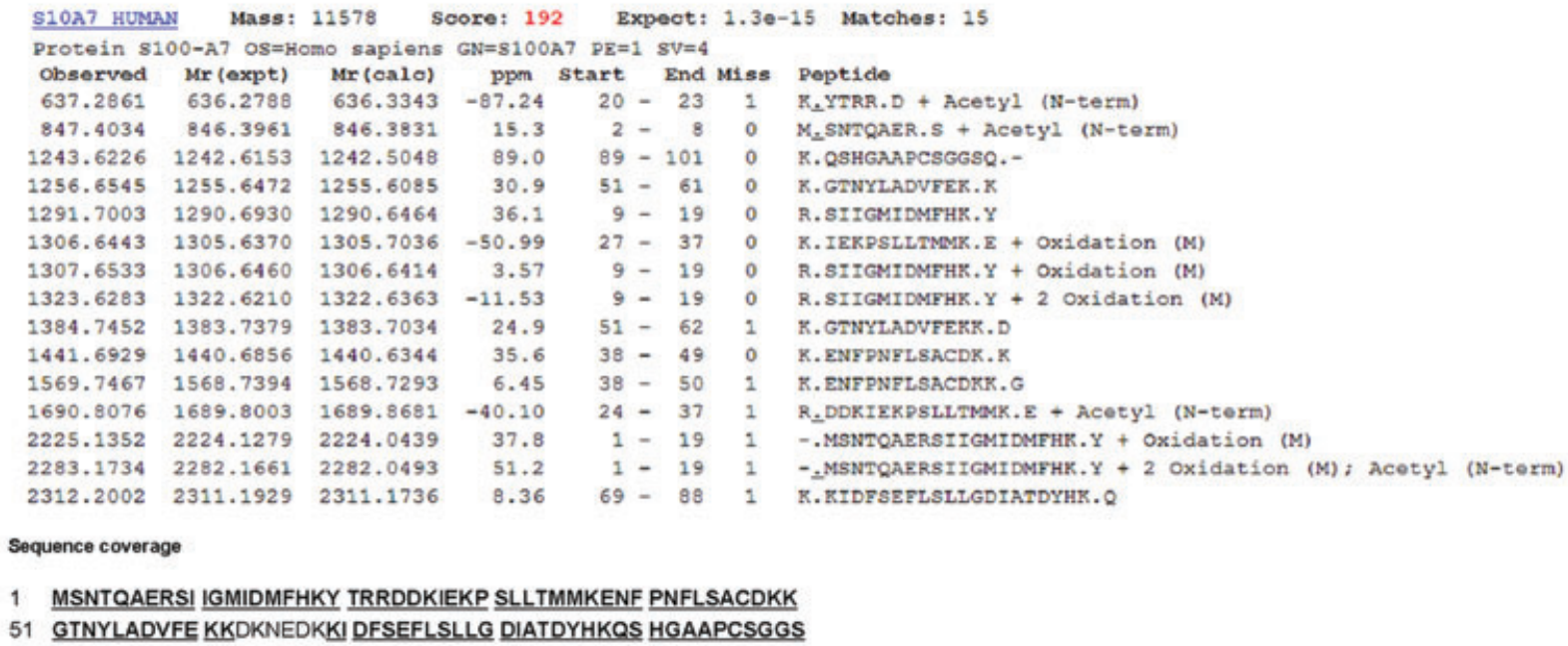

Figure 4. Listing of the most prominent $m / z$ values identified by the MALDI-TOF spectrometry and sequence coverage of the two S100A7a and $b$, respectively.

\subsection{Differential S100A7 occurrence}

The differential expression of the S100A7 forms among the 100 patients was recorded in the matched proteomic maps. The qualitative evaluation revealed the presence of protein spots corresponding to the S100A7a and $b$ in 51 and 63 patients, while they were undetectable in 49 and 37 patients, respectively.

The relative abundance of each isoform was then calculated in the positive cases, following the Image Master algorithms and spot normalization for actin content. According to this methods, we generated three different expression levels, namely low (N\%V 0.01-0.09), medium (N\%V 0.1-0.19), and high $(\mathrm{N} \% \mathrm{~V} \geq 0.2)$. A more detailed description of the S100A7 expression levels is represented in the graph in Fig. 5. As it is possible to observe, while the isoform $a$ is undetectable in $49 \%$ of patients, the isoform $b$ is undetectable in $37 \%$ of patients. Each isoform is expressed at low level in $42 \%$ of patients and at medium expression levels, respectively, in $9 \%$ and $10 \%$. Only isoform $b$ is expressed at high levels in $11 \%$ of patients.

\subsection{Immunolocalization of S100A7}

In consideration of the equivalent immunological reactivity of the two isoforms to the full-length S100A7 antibody (Santa Cruz), we then investigated the tissue localization of 


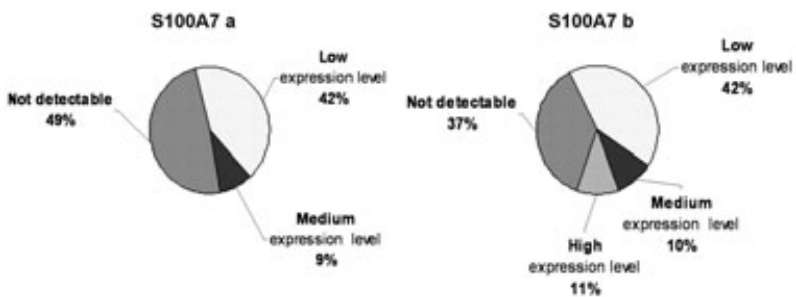

Figure 5. Diagrams reporting the level distributions of the S100A7 among patients. The protein spot expression level was calculated in each map as the \%Vol normalized for actin content. The range of values are expressed on an arbitrary scale, from 0.01 to $>0.2$, and indicated as: low (0.01-0.09), medium (0.1-0.19) and high $(>0.2)$ expression level.
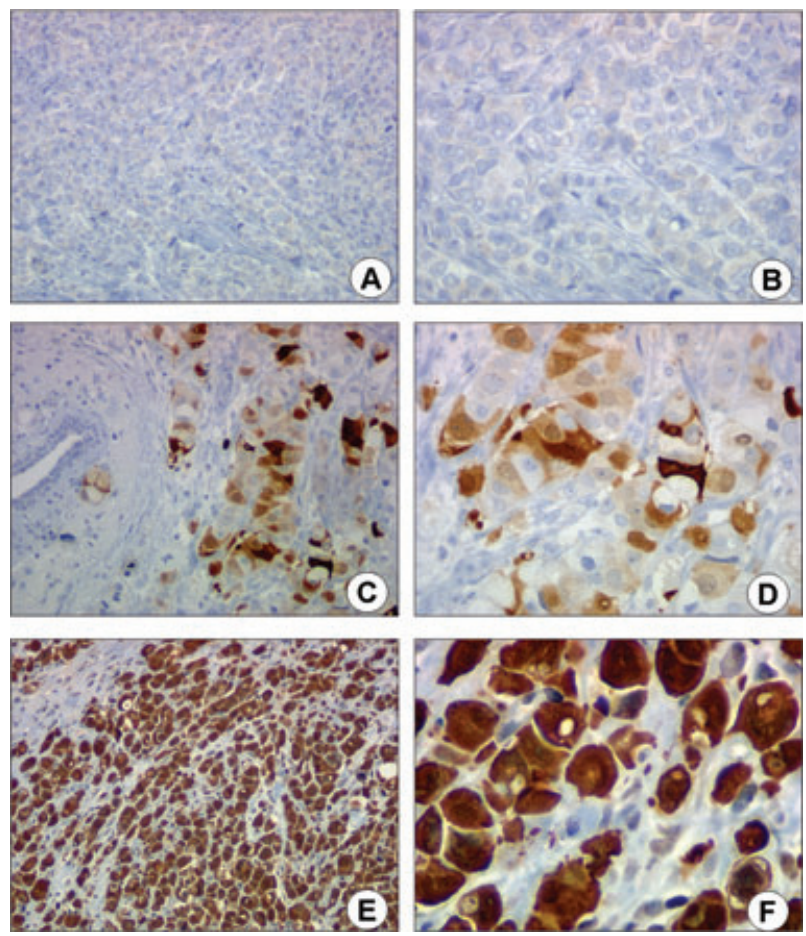

Figure 6. Panel of histoimmunochemical micrographs of three representative cases of IDC, where the reaction to anti-S100A7 antibody was scored as negative $(A / B)$, moderate $(C / D)$, or strong $(\mathrm{E} / \mathrm{F})$. In all cases, the cellular localization is restricted to the neoplastic cells population and absent in the stromal components. In figures, (D-F) is possible to observe both cytoplasmic and nuclear localization of the staining. (A-C-E): original magnification $\times 100$; $(B-D-F)$ : original magnification $\times 200$.

S100A7 on paraffin embedded sections, chosen randomly among tumor explants of our collection. Figure 6 presents a panel of histoimmunochemical micrographs of three representative cases, where the reaction was scored as negative $(A / B)$, moderate $(C / D)$, or strong $(E / F)$. The cellular localization, as it can be observed at low and higher magnification, is clearly restricted to the neoplastic cells population, while the nonaffected ducts and the stromal cells, including en-

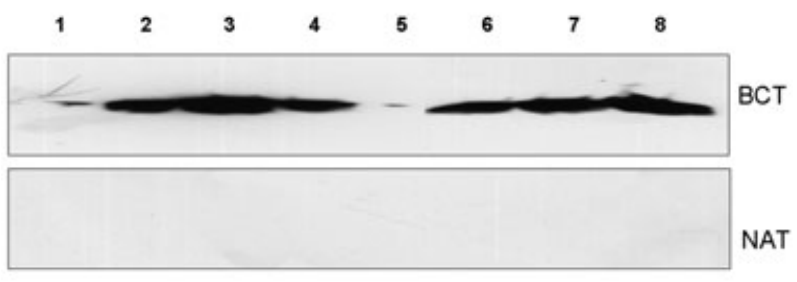

Figure 7. Monodimensional Western blot of S100A7 expression from prototypes of matched breast cancer tissues (BCT) and nontumoral adjacent tissues (NAT).

dothelium, were entirely negative. The sections in figure E/F reveals that the expression of high levels of S100A7 is correlated with an extensive number of neoplastic cells with cytoplasmic and nuclear positivity. Validation of IHC results was performed in tissue extracts. A group of eight tumor samples selected among the ones showing proteomic positivity for S100A7 were compared with their nontumoral paired surgical tissues. Figure 7 shows the Western blot assay performed on monodimensional gels, revealing the unique expression, even at different levels, of the protein in the tumor tissue extracts and its absence in the adjacent nontumoral tissues.

\subsection{Proteomic correlations of S100A7 and functional classification}

Correlation analyses of the relative expression levels of S100A7 isoforms and the protein complement detected in the 100 patients' maps were performed by the graph-pad software using Pearson correlation. A $p$ value less than 0.05 was considered significant, a $p$ value less than 0.01 was considered highly significant and a $p$ value less than 0.001 very highly significant.

Among the 206 protein spots identified in our proteomic maps, 43 (21\% of the total proteins) were significantly correlated with S100A7. Specifically, ten of them were correlated with the more acidic S100A7 form, 37 with the more basic one, while only four (ANXA2 b, ANXA5, one isoform of ENOA, and PRDX6) were correlated with both S100A7 isoforms. Table 1 shows the list corresponding to the S100A7associated proteins.

The functional classification performed by bioinformatic databases resolved the catalogue of S100A7-associated proteins into different clusters trough that S100A7 isoforms may operate with direct or indirect interactions. In particular, the $\mathrm{S} 100 \mathrm{~A} 7 b$ was found related at high score to proteins with prominent cytoplasmic activities and crossing pathways, such as calcium binding (CALM, CALR, S100A2, S100A4, S100A6b, S100A8, S100A11, ANXA2b, ANXA5), signaling and cell motion (GDIS, TPM4, VINC), heat shock and folding (HSP74, PPIA), antioxidant pathways (PRDX1, PRDX6, SODM, GSTP1) metabolic pathways (ACON, ALDR, LDHA, 


\section{Clinical Relevance}

In this study, we describe a large-scale proteomic investigation performed on 100 breast cancer patients for the screening of multiple forms of S100A7 protein. Our present contribution concerns the incidence of S100A7 protein in breast cancer patients and its correlation with the collective profile of cancer patients' proteomics.
We believe that this experimental effort may respond to a present-day challenge of the oncologic research and that may offer a significant contribution to the knowledge and clinical applications of the S100 protein family.
Table 1. S100A7-associated proteins

\begin{tabular}{|c|c|c|}
\hline & S100A7a & $\mathrm{S} 100 \mathrm{~A} 7 b$ \\
\hline ACON & - & $p<0.001^{* * *}$ \\
\hline ALDR a & - & $p<0.01^{* *}$ \\
\hline ALDR b & - & $p<0.05^{*}$ \\
\hline AN32A & - & $p<0.05^{*}$ \\
\hline ANXA2 b & $p<0.05^{*}$ & $p<0.05^{*}$ \\
\hline ANXA5 & $p<0.05^{*}$ & $p<0.05^{*}$ \\
\hline CALM & - & $p<0.01^{* *}$ \\
\hline CALR & - & $p<0.05^{*}$ \\
\hline CATD b & - & $p<0.05^{*}$ \\
\hline EF1B & - & $p<0.05^{*}$ \\
\hline ENOA a & - & $p<0.01^{* *}$ \\
\hline ENOA d & $p<0.05^{*}$ & $p<0.01^{* *}$ \\
\hline G3P b & - & $\mathrm{p}<0.05^{*}$ \\
\hline GDIR & $p<0.001^{* * *}$ & - \\
\hline GDIS & - & $p<0.05^{*}$ \\
\hline GSTP1 & - & $p<0.01^{* *}$ \\
\hline HSP60 b & $p<0.05^{*}$ & - \\
\hline HSP74 & - & $p<0.05^{*}$ \\
\hline LDHA & - & $p<0.05^{*}$ \\
\hline LDHB & - & $p<0.01^{* *}$ \\
\hline LEG1 b & - & $p<0.05^{*}$ \\
\hline NDKB & - & $p<0.01^{* *}$ \\
\hline NTF2 & - & $p<0.01^{* *}$ \\
\hline PHB & - & $p<0.05^{*}$ \\
\hline PPIA c & $p<0.01^{* *}$ & - \\
\hline PPIA e & - & $p<0.05^{*}$ \\
\hline PRDX1 c & - & $p<0.05^{*}$ \\
\hline PRDX6 & $p<0.05^{*}$ & $p<0.05^{*}$ \\
\hline PSA5 & - & $p<0.01^{* *}$ \\
\hline RABP2 & $p<0.01^{* *}$ & - \\
\hline S100A2 & - & $p<0.05^{*}$ \\
\hline S100A4 & - & $p<0.001^{* * *}$ \\
\hline S100A6 b & - & $p<0.05^{*}$ \\
\hline S100A8 & - & $p<0.001^{* * *}$ \\
\hline S100A11 & - & $p<0.01^{* *}$ \\
\hline SODM b & - & $p<0.001^{* * *}$ \\
\hline TAGL2 sf & $p<0.001^{* * *}$ & - \\
\hline TPM4 b & - & $p<0.01^{* *}$ \\
\hline TYPH & - & $p<0.05^{*}$ \\
\hline UBIQ a & $p<0.05^{*}$ & - \\
\hline UBIQ b & - & $p<0.05^{*}$ \\
\hline VINC a & - & $p<0.05^{*}$ \\
\hline VINC b & - & $p<0.05^{*}$ \\
\hline
\end{tabular}

* significant; ${ }^{*}$ highly significant $* *$ very highly significant.
LDHB, ENOA, G3P $b$, TYPH) and regulation of apoptosis (AN32A, ANXA2b, ANXA5, CATD, UBIQ, EF1B, LEG1, PHB, NDKB, NTF2, PSA5).

On the contrary, the S100A7a was correlated with a restricted number of apparently unrelated proteins, six of which also unrelated to the $b$ isoform (GDIR, HSP60, an isoform of PPIA, RABP2, TAGL2 sf, an isoform of UBIQ).

\section{Discussion}

We report here a large-scale proteomic screening of S100A7 calcium-binding protein among a group of 100 patients operated for ductal infiltrating carcinoma of the breast.

The identity of the two isoforms was assessed by mass spectrometry and immunologically confirmed. The two isoelectric forms, arbitrarily named S100A7 $a$ and S100A7b, displayed similar MW and distinct $\mathrm{p} I$; the $\mathrm{S} 100 \mathrm{~A} 7 \mathrm{~b}$ focused in a slightly more basic $\mathrm{p} I$ and appeared more intense than to the S100A7a, usually expressed at low intensity levels. The mass spectrometry analysis showed a sequence coverage with the theoretical masses from the Swiss-Prot sequence database of about $95 \%$ for isoform $b$, and about $60 \%$ for the isoform $a$, in which one of three of the sequence to the carboxy terminus was uncovered by the mass fingerprinting search. Since this portion contains part of functional domains of the S100A7, namely a putative EF-hand domain, part of a putative calcium-binding domain and two zinc-binding residues, it is reasonable to believe that the two isoforms may exert diversified functions in the cell. We think that this is an important contribution to the knowledge of S100A7 isoforms in general, but also for their sporadic occurrence in breast cancer: an open intriguing question that deserves further investigation.

Retrospective immunohistological search on paraffin embedded tumor explants, selected among S100A7 proteomicpositive cases, showed the exclusive localization of the S100A7 immune-reaction within the neoplastic cells, while it was undetectable in the healthy glandular structure and in the surrounding stroma. The absence of clear immunereaction in the nonaffected tissue around the tumor was also confirmed by western blot assays on tissue extracts from the paired nontumoral tissues of the selected cases. 
Several authors have also reported specific expression of S100A7, at both mRNA and protein levels in neoplastic tissues, and its absence in paired noncancerous tissues $[17,32]$, while according to other authors S100A7 is among the most highly expressed genes in preinvasive ductal carcinoma in situ [33], and downregulated with progression to invasive carcinoma $[19,21]$. On the other hand, the persistent overexpression of S100A7 in invasive breast carcinomas is considered a prognostic sign of poor clinical outcome, especially in estrogen receptor-negative invasive ductal carcinomas $[22,23]$. So far, several putative functions have been proposed to explain the involvement of S100A7 protein in breast tumor progression [34]. For instance, some authors have suggested that Psoriasin may support cell invasion, angiogenesis and enhance selection for cells, promoting the upregulation and downregulation of MMP-13 and vascular endothelial growth factor, respectively [35-37]. However, it has not been so far established whether S100A7 contributes to breast cancer growth or metastasis. Our data are the first contribution to the possible role of S100A7 isoforms within a proteome-functional clusterization of S100A7-associated proteins.

A major point of interest is the existence of two distinct isoforms of the S100A7, which correlate at different extent with protein clusters, expressed by the patient's proteomics. The apparently major form, the $b$ isoform, is significantly correlated with at least 33 different proteins involved in critical cellular activities and metabolic pathways. On the contrary, the isoform $a$, which appears as minoritaire for level of abundance and occurrence within patients, is related to a relative small number of proteins apparently unrelated to each others, but with key roles in cancer progression, such as GDIR, TAGL2, and RABP2 [38-40].

As a result of this knowledge, we suggest that these important cellular processes/pathways may be the central mediator in several of the biological circuits through which S100A7 promote tumor progression in breast cancer cells.

All authors participated in interpretation and elaboration of the findings. I.P.M. and P.C. were responsible for the conception and design of the study and drafted the manuscript. G.D.C. performed the protein identification. N.N.A. and P.C. performed protein analysis. F.C., M.R.M., and R.M. carried out 2D-IPG and WB. C.L. performed immunolocalization assay. E.R. and I.R. contributed with clinical informations. All authors read and approved the final manuscript. The present work was supported in part by funds of the Operative Regional Program Sicily 3.14 (DIAMOL project) and by State-made contributions to C.OB.S. $(5 \times 1000)$ for distinguished no-profit research associations.

The authors have declared no conflicts of interest.

\section{References}

[1] Moore, B. W., A soluble protein characteristic of the nervous system. Biochem. Biophys. Res. Commun. 1965, 19, 739-744.
[2] Donato, R., Functional roles of $\mathrm{S} 100$ proteins, calciumbinding proteins of the EF-hand type. Biochim. Biophys. Acta 1999, 3, 191-231.

[3] Deloulme, J. C., Gentil, B. J., Baudier, J., Monitoring of S100 homodimerization and heterodimeric interactions by the yeast two-hybrid system. Microsc. Res. Tech. 2003, 60, 560-568.

[4] Donato, R., S100: a multigenic family of calcium-modulated proteins of the EF-hand type with intracellular and extracellular functional roles. Int. J. Biochem. Cell Biol. 2001, 33, 637-668.

[5] Donato, R., Intracellular and extracellular roles of S100 proteins. Microsc. Res. Tech. 2003, 60, 540-551.

[6] Heizmann, C. W., Fritz, G., Schäfer, B. W., S100 proteins: structure, functions and pathology. Front Biosci. 2002, 7, d1356-d1368.

[7] Leclerc, E., Fritz, G., Vetter, S. W., Heizmann, C. W., Binding of S100 proteins to RAGE: an update. Biochim. Biophys. Acta 2009, 1793, 993-1007.

[8] Santamaria-Kisiel, L., Rintala-Dempsey, A. C., Shaw, G. S., Calcium-dependent and -independent interactions of the S100 protein family. Biochem. J. 2006, 396, 201-214.

[9] Sedaghat, F., Notopoulos, A., S100 protein family and its application in clinical practice. Hippokratia 2008, 12, 198204.

[10] Salama, I., Malone, P. S., Mihaimeed, F., Jones, J. L., A review of the S100 proteins in cancer. Eur. J. Surg. Oncol. 2008, 34, 357-364.

[11] Cancemi, P., Di Cara, G., Albanese, N. N., Costantini, F. et al., Large-scale proteomic identification of S100 proteins in breast cancer tissues. BMC Cancer 2010, 10, 476.

[12] Madsen, P., Rasmussen, H. H., Leffers, H., Honoré, B. et al., Molecular cloning, occurrence, and expression of a novel partially secreted protein "psoriasin" that is highly upregulated in psoriatic skin. J. Invest. Dermatol. 1991, 97, 701712.

[13] Moubayed, N., Weichenthal, M., Harder, J., Wandel, E. et al., Psoriasin (S100A7) is significantly up-regulated in human epithelial skin tumours. J. Cancer Res. Clin. Oncol. 2007, 133, 253-261.

[14] Kesting, M. R., Sudhoff, H., Hasler, R. J., Nieberler, M. et al., Psoriasin (S100A7) up-regulation in oral squamous cell carcinoma and its relation to clinicopathologic features. Oral Oncol. 2009, 45, 731-736.

[15] Ji, J., Zhao, L., Wang, X., Zhou, C. et al., Differential expression of $\mathrm{S} 100$ gene family in human esophageal squamous cell carcinoma. J. Cancer Res. Clin. Oncol. 2004, 130, 480486.

[16] Ostergaard, M., Wolf, H., Orntoft, T. F., Celis, J. E., Psoriasin (S100A7): a putative urinary marker for the follow-up of patients with bladder squamous cell carcinomas. Electrophoresis 1999, 20, 349-354.

[17] Zhang, H., Zhao, Q., Chen, Y., Wang, Y. et al., Selective expression of S100A7 in lung squamous cell carcinomas and large cell carcinomas but not in adenocarcinomas and small cell carcinomas. Thorax 2008, 63, 352-359. 
[18] El-Rifai, W., Moskaluk, C. A., Abdrabbo, M. K., Harper, J., et al., Gastric cancers overexpress S100A calcium-binding proteins. Cancer Res. 2002, 62, 6823-6826.

[19] Leygue, E., Snell, L., Hiller, T., Dotzlaw, H., et al., Differential expression of psoriasin messenger RNA between in situ and invasive human breast carcinoma. Cancer Res. 1996, 56, 4606-4609.

[20] Petersson, S., Bylander, A., Yhr, M., Enerbäck, C., S100A7 (Psoriasin), highly expressed in ductal carcinoma in situ (DCIS), is regulated by IFN-gamma in mammary epithelial cells. BMC Cancer 2007, 7, 205.

[21] Carlsson, H., Petersson, S., Enerbäck, C., Cluster analysis of S100 gene expression and genes correlating to psoriasin (S100A7) expression at different stages of breast cancer development. Int. J. Oncol. 2005, 27, 1473-1481.

[22] Emberley, E. D., Alowami, S., Snell, L., Murphy, L. C., et al., S100A7 (psoriasin) expression is associated with aggressive features and alteration of Jab1 in ductal carcinoma in situ of the breast. Breast Cancer Res. 2004, 6, R308-R315.

[23] Emberley, E. D., Niu, Y., Njue, C., Kliewer, E. V. et al., Psoriasin (S100A7) expression is associated with poor outcome in estrogen receptor-negative invasive breast cancer. Clin. Cancer Res. 2003, 9, 2627-2631.

[24] Jinquan, T., Vorum, H., Larsen, C. G., Madsen, P. et al., Psoriasin: a novel chemotactic protein. Invest. Dermatol. 1996, $107,5-10$.

[25] West, N. R., Watson, P. H., S100A7 (psoriasin) is induced by the proinflammatory cytokines oncostatin- $\mathrm{M}$ and interleukin-6 in human breast cancer. Oncogene 2010, 29, 2083-2092.

[26] Emberley, E. D., Gietz, R. D., Campbell, J. D., HayGlass, K. T. et al., RanBPM interacts with psoriasin in vitro and their expression correlates with specific clinical features in vivo in breast cancer. BMC Cancer 2002, 2, 28.

[27] Ruse, M., Broome, A. M., Eckert, R. L., S100A7 (psoriasin) interacts with epidermal fatty acid binding protein and localizes in focal adhesion-like structures in cultured keratinocytes. J. Invest. Dermatol. 2003, 121, 132141.

[28] Emberley, E. D., Niu, Y., Leygue, E., Tomes, L. et al., Psoriasin interacts with Jab1 and influences breast cancer progression. Cancer Res. 2003, 63, 1954-1961.

[29] Pucci-Minafra, I., Cancemi, P., Albanese, N. N., Di Cara, G. et al., New protein clustering of breast cancer tissue pro- teomics using actin content as a cellularity indicator. J. Proteome Res. 2008, 7, 1412-1418.

[30] Saeed, A. I., Bhagabati, N. K., Braisted, J. C., Liang, W. et al., TM4 microarray software suite. Methods Enzymol. 2006, 411, 134-193.

[31] Pucci-Minafra, I., Fontana, S., Cancemi, P., Alaimo, G., et al., Proteomic patterns of cultured breast cancer cells and epithelial mammary cells. Ann. NY Acad. Sci. 2002, 963, 122139.

[32] Pucci-Minafra, I., Cancemi, P., Fontana, S., Minafra, L. et al., Expanding the protein catalogue in the proteome reference map of human breast cancer cells. Proteomics 2006, 6, 26092625.

[33] Jiang, W. G., Watkins, G., Douglas-Jones, A., Mansel, R. E., Psoriasin is aberrantly expressed in human breast cancer and is related to clinical outcomes. Int. J. Oncol. 2004, 25, 81-85.

[34] Porter, D. A., Krop, I. E., Nasser, S., Sgroi, D. et al., A SAGE (serial analysis of gene expression) view of breast tumor progression. Cancer Res. 2001, 61, 5697-5702.

[35] Emberley, E. D., Murphy, L. C., Watson, P. H., S100A7 and the progression of breast cancer. Breast Cancer Res. 2004, 6, 153-159.

[36] Morgan, M. R., Jazayeri, M., Ramsay, A. G., Thomas, G. J., et al., Psoriasin (S100A7) associates with integrin $\beta 6$ subunit and is required for $\alpha \mathrm{V} \beta 6$-dependent carcinoma cell invasion. Oncogene 2011, 30, 1422-1435.

[37] Paruchuri, V., Prasad, A., McHugh, K., Bhat, H. K. et al., S100A7-downregulation inhibits epidermal growth factorinduced signaling in breast cancer cells and blocks osteoclast formation. PLoS One 2008, 3, e1741.

[38] Liang, Z., Hui, W., Jianming, L., Yawei, L., et al., Overexpression of Rho GDP-dissociation inhibitor alpha is associated with tumor progression and poor prognosis of colorectal cancer. J. Proteome Res. 2008, 7, 3994-4003.

[39] Zhang, Y., Ye, Y., Shen, D., Jiang, K. et al., Identification of transgelin-2 as a biomarker of colorectal cancer by laser capture microdissection and quantitative proteome analysis. Cancer Sci. 2010, 101, 523-529.

[40] Budhu, A. S., Noy, N., Direct channeling of retinoic acid between cellular retinoic acid-binding protein II and retinoic acid receptor sensitizes mammary carcinoma cells to retinoic acid-induced growth arrest. Mol. Cell. Biol. 2002, 22, 2632-2641. 